\title{
Assessment of Patient Education about Statin Therapy on Quality Measures and Knowledge in an Independent Community Pharmacy
}

Sean W. Clark, PharmD $D^{1}$; Gretchen K. Garofoli, PharmD, BCACP2; Betsy M. Elswick, PharmD

${ }^{1}$ PGY-1 Community-Based Pharmacy Resident at West Virginia University School of Pharmacy, Waterfront Family Pharmacy

${ }^{2}$ Associate Professor at West Virginia School of Pharmacy in the Department of Clinical Pharmacy, Waterfront Family Pharmacy

${ }^{3}$ Associate Professor and Residency Program Director, PGY-1 Community-Based Residency Program, West Virginia University School of Pharmacy

\begin{abstract}
Objectives: The objectives were to 1) assess the possible impact of face-to-face patient education on Electronic Quality Improvement Platform for Plans and Pharmacies (EQuIPP) performance scores, 2) determine if face-to-face patient education increased overall knowledge and number of identified patients on statin therapy, and 3) identify barriers to statin therapy in targeted patients with diabetes.

Design: Participants received an anonymous survey tool collecting demographic data and assessing barriers, baseline knowledge, and perceptions about statin therapy. Following the initial survey, participants received education from the pharmacist describing the risks and benefits of statin therapy and were given a supplemental pamphlet. A second post-education survey tool was given to assess posteducation knowledge and perceptions. At the end of the study period, investigators assessed the number of participants started on statin therapy and calculated the predicted percentage change in EQuIPP score.

Setting and Participants: This study was conducted at Waterfront Family Pharmacy in Morgantown, West Virginia from December 2017 until April 2018. Participants were included if aged 40 to 75, received at least two fills of a diabetes medication at the pharmacy in the last year, had not taking a statin within a year prior to participating in the study, and could read and write in English.

Outcome Measures: The primary outcome measure was the predicted percentage change in the "Statin Use in Diabetes" EQuIPP Score. Secondary measures included post-educational knowledge and perceptions of statin therapy.

Results: During the study period, 10 participants completed the surveys and educational intervention. The predicted change in "Statin Use in Diabetes" EQuIPP score was an increase from $75 \%$ to $76.9 \%$ (+ 1.9\%). Prior to the educational intervention, none of the participants could identify a benefit of statin therapy aside from lowering cholesterol. After the intervention, $80 \%$ of participants could identify at least one additional benefit of statin therapy. Before the intervention, $30 \%$ of participants stated they would consider taking a statin, which increased to $80 \%$ following the pharmacist-led education. Lastly, no participants felt they needed to be on statin therapy prior to the intervention. Following the intervention, $40 \%$ stated they believed they were candidates for statin therapy.

Conclusions: Patients are willing to receive education from pharmacists about their medications and are receptive to general recommendations. A common modifiable barrier to statin therapy is patient knowledge, emphasizing the importance of pharmacistprovided education. Education about statin therapy may also increase EQuIPP scores in an independent community pharmacy, leading to better outcomes for patients and improvement of common performance measures. Overall, it appears patients require more education about statin therapy and the benefit these drugs can provide aside from their cholesterol lowering properties. Pharmacistprovided education regarding statins in patients with diabetes can increase performance measures monitored by third party payers.
\end{abstract}

Key Words: Statin, Education, Diabetes, Quality Measures

\section{Background}

Diabetes is a chronic, metabolic disease characterized by elevated levels of blood glucose, which can lead to serious damage of the heart, blood vessels, eyes, kidneys, and nerves. Globally, the number of people with diabetes has risen from 108 million in 1980 to 422 million in 2014, and the World Health Organization projects that diabetes will be the seventh leading cause of death by $2030 .^{1}$

Corresponding author: Sean W. Clark, PharmD West Virginia University School of Pharmacy Waterfront Family Pharmacy, Morgantown, WV Email: swclark@mix.wvu.edu
HMG-CoA reductase inhibitors, also known as statins, are proven to reduce the risk of cardiovascular events in patients with diabetes. Because of this proven benefit, the American Diabetes Association and American Heart Association/American College of Cardiology have strong recommendations regarding statin therapy. These organizations recommend that most patients with diabetes, between the age of 40 and 75 and with no contraindications, be started on at least moderate intensity statin therapy. ${ }^{2-4}$

Despite the proven benefit of statin therapy and recommendations by expert panels, its use in patients with diabetes remains low. According to the National Diabetes Statistics Report, $41.8 \%$ of adults aged 21 years or older with no self-reported cardiovascular disease, but who were eligible for statin therapy, were not on a lipid-lowering medication. For 
adults aged 21 years or older with self-reported cardiovascular disease, $33.1 \%$ were not on lipid-lowering medication. ${ }^{5}$ This gap in care leaves a large proportion of patients with diabetes at risk for the development, or progression, of cardiovascular disease.

The incidence of patients with diabetes not taking statin therapy could be attributed to several factors including therapy intolerance, contraindications, or deficits in knowledge. One study showed that patients have a lack of perceived benefits and underestimate their susceptibility to dyslipidemia-related complications. ${ }^{6}$ Based on this information, it appears that rates of statin use in patients with diabetes can be improved through effective patient education programs.

Third party payers, including the Centers for Medicare and Medicaid Services (CMS), also focus on this patient population. CMS uses a Star Rating System to measure how well Medicare Advantage and Part D plans perform. Medicare evaluates how plans perform in several categories, including "Statin Use in Diabetes." A plan's performance rating is based on the sum of its providers, including pharmacies. Pharmacies are not assigned Star Ratings, but those that improve medication use and a plan's Star Ratings can see benefits such as preferred pharmacy network status, leading to potential increases in revenue. Pharmacies can monitor the status of the Star Rating quality measures through the Electronic Quality Improvement Platform for Plans and Pharmacies (EQulPP). EQulPP allows pharmacies to track Medicare outcome measures and identify areas in need of improvement. The measure of "Statin Use in Diabetes" provides pharmacies with the percentage of Medicare patients with diabetes who are taking a statin. An EQuIPP score of $80.3 \%$ or higher indicates pharmacies are meeting the requirements defined by Medicare. ${ }^{7-8}$ Using these tools, pharmacists can work to optimize medication therapy and improve cardiovascular outcomes in patients with diabetes.

\section{Objectives}

The objectives of this study were to assess the possible impact of face-to-face patient education on EQuIPP performance scores, determine if face-to-face patient education increased overall knowledge and number of identified patients on statin therapy, and identify barriers to statin therapy in targeted patients with diabetes.

\section{Methods}

This prospective targeted intervention study used a patient engagement software program called PrescribeWellness ${ }^{\odot}$ to identify potential participants at Waterfront Family Pharmacy in Morgantown, WV. This study was approved by the West Virginia University Institutional Review Board. This system identified all eligible participants, regardless of prescription coverage, and included those without Medicare. Participants were approached by investigators at the pharmacy counter when picking up a prescription or contacted via phone to inform them of the project and assess their interest in participation. Investigators also followed up with potential participants via phone if they were unable to be reached during the initial encounter. Data collection started on December $1^{\text {st }}, 2017$ and continued until April $30^{\text {th }}, 2018$. Participants between the ages of 40 and 75 were included if they received at least two fills of a diabetes medication at the pharmacy in the last year, had not taken a statin within a year prior to being enrolled into the study, and could read and write in English. Participants were excluded if their age was less than 40 or over 75 , could not read or write in English, or had any apparent contraindication to statin therapy.

An anonymous written pre-survey (Appendix A) was used to determine demographic data, health history, baseline knowledge about statins, previous statin use, willingness to start a statin, and perceptions about therapy. After completion of the initial survey, participants received education from the project's primary investigator describing the risks and benefits of statin therapy. This education was consistent among participants, and based on a patient education pamphlet (Appendix B) created by investigators. After receiving education, participants were also given a written pamphlet containing the information that was discussed. After the educational intervention, participants received a second anonymous survey (Appendix C) to assess post-education knowledge about statin therapy, willingness to start therapy, and their perceptions about therapy. Following the study period, investigators assessed the number of participants that had received and filled a new prescription for a statin medication. Investigators also calculated the predicted percentage change in the "Statin Use in Diabetes" EQulPP score and compiled survey responses. The predicted percentage change in EQuIPP scores was calculated instead of the actual change. This method was chosen because EQuIPP data reporting lags approximately 2 months behind data collection and is reported as a 6-month or year-to-date average. It would also be difficult for investigators to account for patients who transferred a prescription into or out of the pharmacy, which could affect the overall score. The score was calculated using the following equation:

Predicated $\%$ Change $=$ Total \# of Medicare participants at begining of study period
Minus
Total \# of Medicare participants at begining of study period

\section{Results}

Prior to the study period, 56 participants were identified for inclusion. Of these participants, 10 completed the surveys and educational intervention resulting in a $17.8 \%$ response rate. The second attempt was completed prior to the end of the study, but was unsuccessful due to lack of interest or inability to make contact. Demographic data collected were as follows; 
average age of 66 years, 8 identified as male, and 10 identified as Caucasian, having at least a high school education/GED, and having a diagnosis of diabetes.

The "Statin Use in Diabetes" EQuIPP score is reported as a percentage. This score calculates the number of patients with Medicare and diabetes that have filled a prescription for a statin versus the number that have not. A total of 52 participants were included in the "Statin Use in Diabetes" EQulPP score calculation. This differs from the original 56 identified participants because four were not covered by Medicare. Of the 52 identified participants with Medicare, 13 were not taking a statin which resulted in an overall EQuIPP score of $75 \%$ at the beginning of December 2017. The predicted percentage change in this EQuIPP score was calculated based on the number of participants with Medicare taking a statin at the start of the study period, versus the number at the end of the study period. At the conclusion of this study, two participants were started on statin therapy. Of these participants, only one had Medicare and was included in the predicted percentage change in EQuIPP score. The predicted percentage change in the "Statin Use in Diabetes" EQuIPP score was $+1.9 \%$ (75\% to $76.9 \%$ ).

The overall intervention, including surveys and education, took an average of 18 minutes per participant. Prior to the educational intervention, participant knowledge and background information about statin therapy was assessed. This assessment found that; $70 \%$ of participants had heard of a medication called a statin, $30 \%$ had spoken to a healthcare provider about statin therapy, $80 \%$ preferred to learn about statins from a doctor or pharmacist, and $30 \%$ had taken a statin previously and discontinued therapy due to side effects. When participants were asked to identify common indications for statin therapy, a gap in knowledge was observed. This gap in knowledge appeared to improve after the educational intervention. Patient perceptions also changed after the educational intervention and showed a notable shift. These changes in knowledge and perceptions are summarized in Figure 1.

\section{Discussion}

This study showed that, despite the proven benefit of statins reducing the risk of cardiovascular events in patients with diabetes ${ }^{2-4}$, there was still a need to provide education regarding therapy. The majority of participants had heard of a statin, but did not know it could be used for more than lowering cholesterol levels. This represented a gap in knowledge which may have led to omission of therapy. This gap represents a modifiable factor that could be focused on to reduce the risk of cardiovascular events in patients with diabetes. This study showed that a brief educational intervention may lead to increased patient knowledge, change in perceptions, and an increase in the number of patients with diabetes on statin therapy.
Platforms like EQuIPP can help pharmacists identify patients with opportunities for improved care. This study showed that educating patients about statin therapy could increase quality outcome measures reported by EQuIPP. Even a small increase in the number of patients meeting the outcome measure could have a large impact on the overall score. Improvement of these measures could lead to more comprehensive care and a possible increase in revenue through meeting the quality outcome measures tracked by third party payers.

Limitations to this study included the use of a non-validated survey and small convenience based sample. The results of this study may not be applicable to patients with contraindications or intolerances to statin therapy. A low response rate was an additional study limitation. Of the 56 participants identified, only 10 participated in the study. Of the participants that did not participate, 32 declined to due to time constraints or lack of interest, and 14 could not be reached. New or lost patients were not factored into the predicted EQuIPP score which could influence the actual score. This study used an educational intervention only and did not attempt to contact prescribers regarding statin therapy, which may have resulted in lower rates of newly initiated statins. This study also lacked generalizability to all areas of the United States since it was conducted at a single site, all participants identified as Caucasian, and a majority identified as male.

Future studies should examine the effect of contacting providers as part of the intervention. These studies may also attempt to determine how to increase patient response rates to a face-to-face interaction, or create a more convenient method of contacting patients outside of face-to-face interactions. Lastly, future studies should attempt to identify alternative ways to improve patient education about statin therapy and assess knowledge gained.

\section{Conclusion}

In conclusion, this study identified a modifiable factor that may prevent patients from utilizing a therapy proven to reduce or prevent cardiovascular disease associated with diabetes. Pharmacist-provided education is well received by patients and can have a positive influence on knowledge and perceptions, leading to increased rates of statin use in patients with diabetes. Providing education may also have a positive impact on quality outcomes measures tracked by third-party payers through platforms like EQuIPP. Improvement of these scores can help to optimize patient care and potentially increase a pharmacy's revenue by becoming a preferred provider in a third-party payer's network. 
Conflicts of interest: We declare no conflicts of interest or financial interests that the authors or members of their immediate families have in any product or service discussed in the manuscript, including grants (pending or received), employment, gifts, stock holdings or options, honoraria, consultancies, expert testimony, patents, or royalties.

Treatment of Human Subjects: IRB review/approval required and obtained.

\section{References}

1. Mathers CD, Loncar D. Projections of Global Mortality and Burden of Disease from 2002 to 2030. Samet J, ed. PLoS Medicine. 2006;3(11):442. doi:10.1371/journal.pmed.0030442.

2. Chou R, Dana T, Blazina I, Daeges M, Jeanne TL. Statins for prevention of cardiovascular disease in adults: evidence report and systematic review for the US preventive services task force. JAMA. 2016;316:2008-2024. doi:10.1001/jama.2015.15629.

3. American Diabetes Association. 9. Cardiovascular Disease and Risk Management: Standards of Medical Care in Diabetes-2018. Diabetes Care. 2018;41:S86S104. doi: 10.2337/dc18-S009.

4. Stone N.J., Robinson J.G., Lichtenstein A.H. 2013 ACC/AHA guideline on the treatment of blood cholesterol to reduce atherosclerotic cardiovascular risk in adults: a report of the American college of cardiology/American heart association task force on practice guidelines. J. Am. Coll. Cardiol. 2014;63:2889-2934. doi: 10.1016/j.jacc.2013.11.002

5. American Diabetes Association. National Diabetes Statistics Report, 2017. Diabetes.Org, 2018. Available at: www.diabetes.org/assets/pdfs/basics/cdcstatistics-report-2017.pdf. Accessed May 23, 2018.

6. Chee YJ, Chan HHV, Tan NC. Understanding patients' perspective of statin therapy: can we design a better approach to the management of dyslipidaemia? A literature review. Singapore Medical Journal. 2014;55(8):416-421. doi:10.11622/smedj.2014099

7. Academy of Managed Care Pharmacy. American Pharmacists Association Medicare star ratings: Stakeholder proceedings on community pharmacy and managed care partnerships in quality. J. Am. Pharm. Assoc. 2014;54:228-240. doi: 10.1331/JAPhA.2014.13180

8. PL Detail-Document, Quality Measures for Pharmacies. Pharmacist's Letter/Prescriber's Letter. January 2016. 
Figure 1. Pre-Educational versus Post-Educational Knowledge/Perceptions about Statin Therapy $(n=10)$

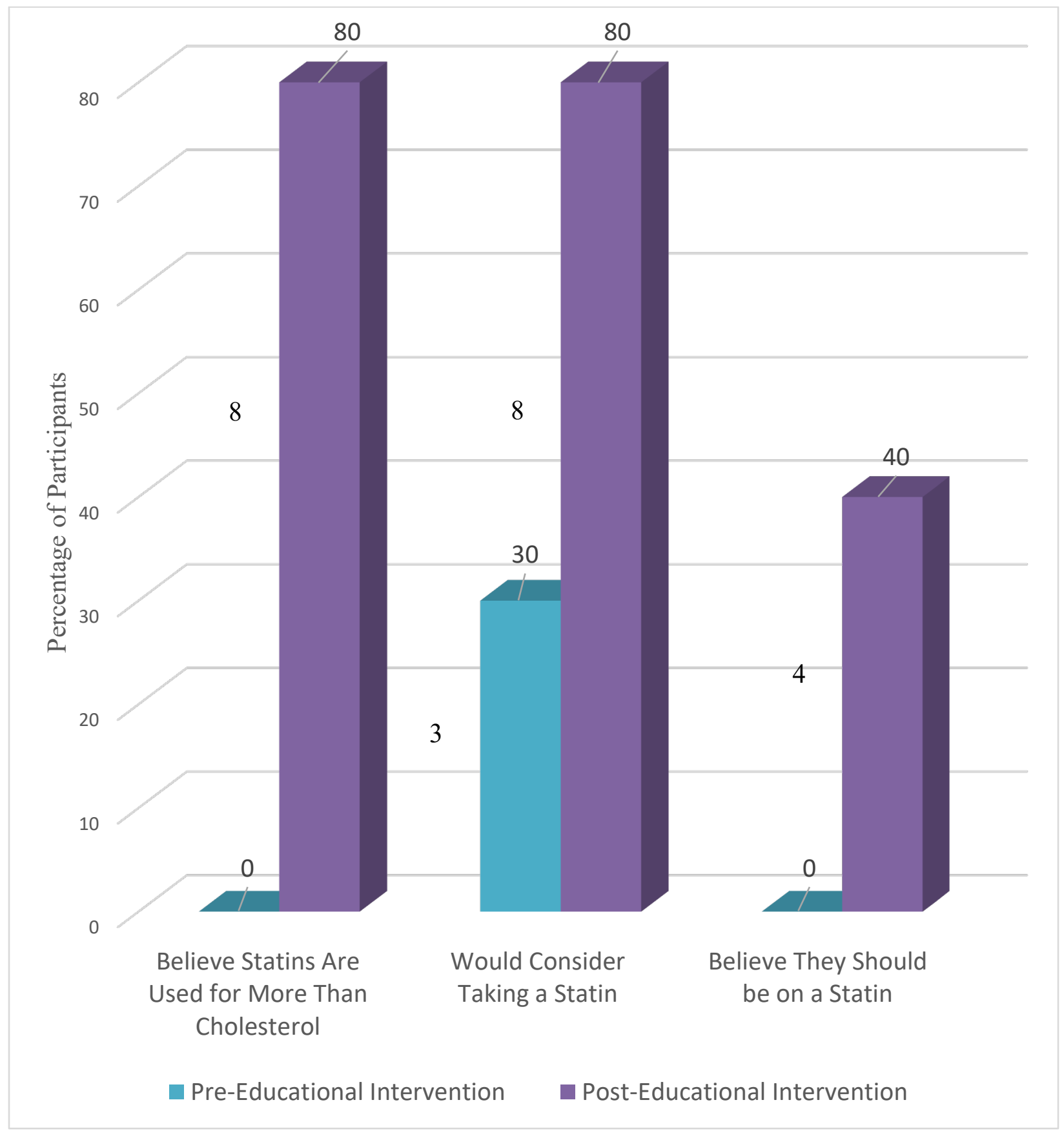




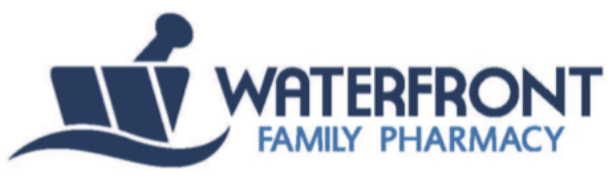

\section{Baseline Statin Survey}

Many patients take cholesterol lowering medications called hydroxymethylglutaryl CoA (HMG CoA) reductase inhibitors, also called "statins." Examples of "statin" medications include Lipitor $^{\circ}$ (atorvastatin), Zocor $^{\circ}$ (simvastatin), Crestor $^{\circ}$ (rosuvastatin), Pravachol ${ }^{\circ}$ (pravastatin), and others. This survey asks about some background information and your thoughts and feelings about taking "statin" medications.

1. What is your age?

2. What is your gender?
a. Male

b. Female

3. What is your ethnicity/race? (Circle one or more)
a. American Indian/Alaska Native
b. Asian
c. African American
d. Native Hawaiian/Other Pacific Islander
e. White
f. Other; please specify:

4. What is your highest level of education?
a. Grade School (0-8)
b. Some High School
c. High School Diploma/GED
d. Some College
e. 2-year College Degree
f. 4-year College Degree
g. Graduate Degree (e.g. MS, PhD)

5. Have you ever been diagnosed with diabetes or told that you have "high sugars"?
a. Yes
b. No

6. Have you ever been diagnosed with liver disease or told that you have problems with your liver?
a. Yes
b. No

7. Have you ever heard of a medication called a statin? e.g. Lipitor ${ }^{\circ}$ (atorvastatin), Zocor ${ }^{\circ}$ (simvastatin), Crestor $^{\circ}$ (rosuvastatin), Pravachol $^{\circ}$ (pravastatin)
a. Yes
b. No 
8. Has your doctor, pharmacist, or another healthcare provider ever talked to you about statins?
a. Yes
b. No
c. I'm not sure

9. Have you ever taken a statin?
a. Yes
b. No
c. I'm not sure

10. If you answered "Yes" to question number 9, are you currently still taking a statin?
a. Yes
b. No; please specify why:
c. I'm not sure

11. Would you ever consider taking a statin if you haven't before?
a. Yes
b. No
c. I'm not sure

12. If you answered "No" to question number 11, Why wouldn't you consider taking a statin? (Circle all that apply)
a. I've heard they are unsafe
b. I don't think I would benefit from them
c. I don't have high cholesterol
d. I know someone who had an adverse reaction to them
e. I don't know enough about them
f. They cost too much
g. Other (please list):

13. What do you think statins are used for? (Circle all that apply)
a. High cholesterol
b. Heart health
c. Preventing strokes
d. High blood pressure
e. Other (please list):

14. How would you prefer to learn about statins?
a. Your Doctor
b. Your Pharmacist
c. Another healthcare professional
d. No preference
e. Other (please list):

15. Do you think you should be on a statin?
a. Yes
b. No
c. I'm not sure 


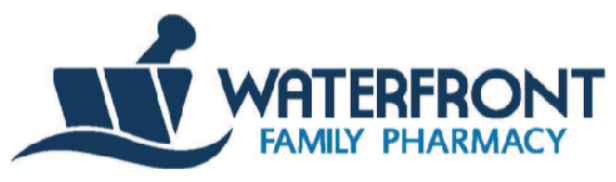

Follow-up Statin Survey

Many patients take cholesterol lowering medications called hydroxymethylghtaryl CoA (HMG CoA) reductase in hibitors, also called "statins." Examples of "statin" medications include Lipitor " (atorvastatin), Zocor ${ }^{\circ}$ (simvastatin), Crestor ${ }^{\circ}$ (rosuvastatin), Pravachol ${ }^{\circ}$ (pravastatin), and others. This survey ask about your thoughts and feelings about taking statin medications after learning more about them.

1. What do you think statins are used for? (Circle all that apply)
a. High cholestero
b. Heart health
c. Preventing strokes
d. High blood pressime

2. After leaming more, would you consider taking a statin medication?
a. Yes
b. No
c. I'm not sure

3. If you answered "No" to question number 2 , why wouldn't you consider taking a statin?
a. I don't think I would benefit from them
b. I still don't think they're safe
c. I would like to leam more
d. Other (please list):

4. Would you consider talking to your doctor about starting a statin?
a. Yes
b. No
c. I'm not sure

5. Would you prefer to have your phammacist contact your doctor about starting a statin?
a. Yes
b. No
c. I'm not sure

6. Do you think you would benefit from starting a statin?
a. Yes
b. No
c. I'm not sure

7. Do you think you should be on a statin?
a. Yes
b. No 


\section{Appendix C}

\section{References}

1. Marathe, P. H., Gao, H. X. and Close, K. L. (2017), American Diabetes Association Standards of Medical Care in Diabetes 2017. Journal of Diabetes, 9: 320-324. doi:10.1111/1753-0407.12524

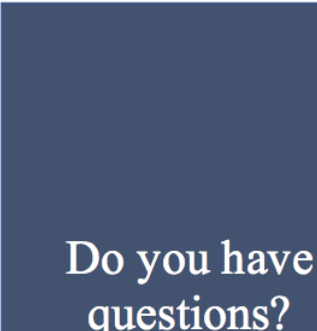

Atoryation. 2017. Available at:

2017. Available at: https://www.upto

information?source=search result\&search=atorvastatin \&selectedTitle=3 126. Accessed October 1, 2017. \&selectedTitle $=3 \sim 126$. Accessed October $1,2017$.
Pravastatin Patient Drug Information. Uptodatecom. 2017. Available at:

2017. Available at: itents/pravastatin-patientdrug-

information?source=search result\&search=pravastatin \&selectedTitle $=3 \sim 126$. Accessed October 1, 2017

4. Rosuvastatin Patient Drug Information. Uptodatecom 2017. Available at:

https://www.uptodate.com/contents/rosuvastatinpatient-drug-

information?source=search result\&search=rosuvastatin \&selectedTitle=3 126. Accessed October 1, 2017

5. Simvastatin Patient Drug Information. Uptodatecom 2017. Available at:

https://www.uptodate.com/contents/simvastatin-patientdrug-

information?source=search_result\&search $=$ simvastatin \&selectedTitle=3 126. Accessed October 1, 2017.

We have answers!
6. Statin side effects: Weigh the benefits and risks. Mayo Clinic. 2017. Available at:

https://www.mayoclinic.org/diseases-conditions/highblood-cholesterol/in-depth/statin-side-effects/art20046013. Accessed October 1, 2017.
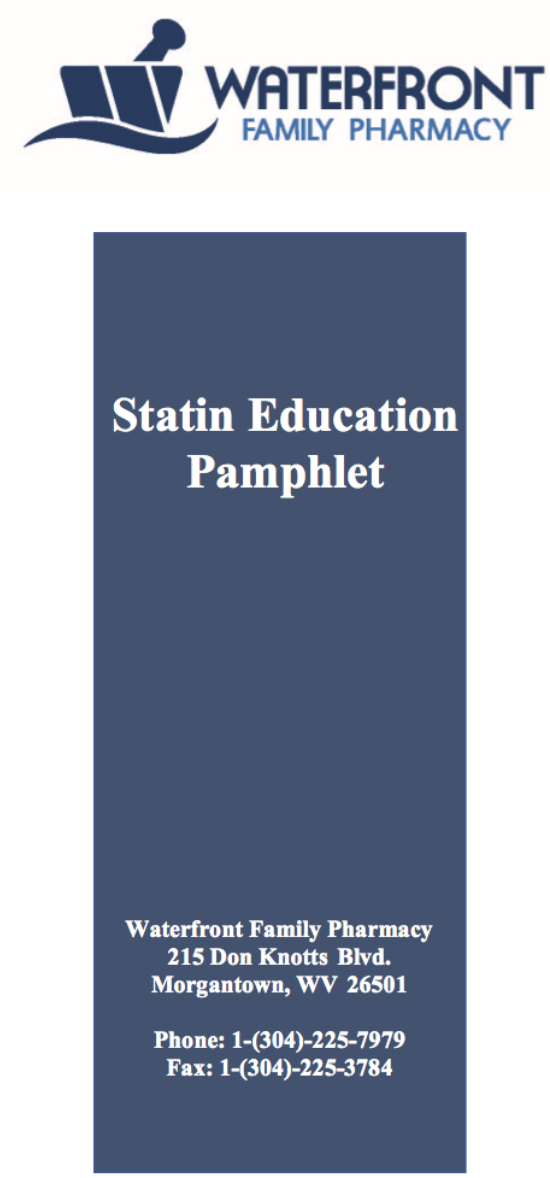


\section{What Are Stains?}

- Statins are another name for hydroxymethylglutaryl CoA (HMG

$\mathrm{CoA}$ ) reductase inhibitors

- Statins are drugs used to:

- Lower "bad" cholesterol

- Lower triglycerides

- Increase "good" cholesterol

- Lowering "bad" cholesterol can prevent the buildup of fatty deposits in your blood vessels that can lead to blockages

- Preventing blockages reduces the risk of heart attacks and strokes

- There are several different statins available, including:

- Simvastatin (Zocor)

- Atorvastatin (Lipitor)

- Rosuvastatin (Crestor)

- Pravastatin (Pravachol)

- Pitavastatin (Livalo)

- Lovastatin (Mevacor)

- Talk with your doctor or pharmacist to determine the best statin for you

\section{Who Can Benefit From Statins}

- Patients with an increased risk of heart disease or stroke, including:

- Patients with high cholesterol

- Patients with diabetes

- Patients with high blood pressure

- Patients who smoke

- Patients who have already had a heart attack or a stroke
- Statins are generally well tolerated

- All drugs have the potential to cause side effects. However, many people have no side effects or only have minor side effects

- Common side effects include:

○ Stomach upset

- Heartburn

○ Diarrhea or constipation

- Headache

- A physician should be contacted if these side effects are severe or don't go away

- Some more serious, but less common side effects include:

- Excessive tiredness

- Yellowing of the skin or eyes

- New or worsening muscle pain, aches, cramping, or weakness

- Problems passing urine

- Dark-colored urine or pale stools

- Rash or itching

- Severe stomach pain

- Chest pain

o Confusion, forgetfulness, or memory loss

- Your doctor should be contacted right away if you have any of these side effects

- Some people should avoid taking a statin if they have:

- Had a severe allergic reaction to a statin in the past

- Have been unable to tolerate statins in the past

- Have a history of liver, kidney, or thyroid disease

- Are pregnant or breastfeeding
- Your doctor will check your bloodwork to make sure the statins are working safely

- Be sure to inform your doctor and pharmacy of all medications you are taking, including over the counter and herbal medications, before starting a statin

- These medications should be use along with diet and exercise to further reduce the risk of heart disease and stroke

- Avoid drinking grapefruit juice or eating grapefruit often, because this can increase statin levels and the risk of side effects

- Avoid or limit drinking alcohol to less than 3 drinks a day. Drinking too much alcohol may raise your chance of liver disease

- This drug may affect how much of some other drugs are in your body

- If you are 65 or older, use this drug with care. You could have more side effects

- It is possible to try a different statin or lower dose if you had a side effect to a statin in the past that wasn't severe or life threatening 